\title{
ENDOSCOPIC IMAGE OF INTEREST
}

\section{SMALL BOWEL PSEUDOMELANOSIS AND ORAL IRON THERAPY}

\author{
Nuno Almeida, ${ }^{1}$ Pedro Figueiredo, ${ }^{1}$ Sandra lopes, ${ }^{1}$ Paulo Freire, ${ }^{1}$ Vítor Sousa, ${ }^{2}$ Clotilde Lérias, ${ }^{1}$ \\ Hermano Gouveia ${ }^{1}$ and Maximino C. Leitão ${ }^{1}$ \\ Departments of ${ }^{1}$ Gastroenterology, ${ }^{2}$ Pathology, Coimbra University Hospital, Coimbra, Portugal
}

\begin{abstract}
Small bowel pseudomelanosis is a rarely reported clinical entity characterized by brown pigmentation of small bowel mucosa. The authors describe two cases, both with iron deficiency anemia, one of an 81-year-old female patient submitted for capsule endoscopy that revealed a brown pigmentation of all small bowel mucosa and another of an 81-year-old male whose retrograde double-balloon enteroscopy revealed a diffuse brown pattern of small bowel mucosa. Ileal biopsies confirmed intense iron deposition in the macrophages of the lamina propria. Both patients were on oral iron therapy and the second one had a previous double-balloon enteroscopy, 2 years earlier, which revealed only ileal angiodysplasias. These two cases demonstrate the importance of two new endoscopic methods for diagnosis of small bowel pseudomelanosis, the rarity of such an entity and its close relation with oral iron therapy.
\end{abstract}

Key words: capsule endoscopy, double-balloon enteroscopy, oral iron therapy, pseudomelanosis, small bowel.

\section{INTRODUCTION}

A dark pigmentation of gastrointestinal mucosa is called pseudomelanosis. Pseudomelanosis coli is common but small bowel pseudomelanosis is rarely reported and apparently associated with oral iron intake. We describe two cases of small bowel pseudomelanosis diagnosed by capsule (CE) and double balloon enteroscopy (DBE) in patients on oral iron therapy.

\section{CASE REPORT}

The first case is of an 81-year-old woman with persistent iron-deficiency anemia, with no macroscopic evidence of overt gastrointestinal bleeding and negative findings on upper and lower endoscopies. She had also hypertension and was medicated with indapamide, dipyridamole and iron sulfate pills (288 mg daily). CE showed a brown pigmentation of the entire small bowel mucosa, in a speckled, continuous pattern (Fig. 1) highly suggestive of small bowel pseudomelanosis.

The second case is of an 81-year-old man, also with irondeficiency anemia. He was medicated with iron sulfate (288 mg daily), lisinopril, furosemide and aminophylline because of hypertension and chronic obstructive pulmonary disease (COPD). In February of 2006, a CE revealed multiple ileal angiodysplasias, successfully treated with argon-plasma coagulation during a retrograde DBE. No other mucosal abnormalities were detected. In February of 2008, anemia recurred and now, a second DBE revealed a speckled pattern of brown pigmentation in the ileum (Fig. 2). Biopsy speci-

Correspondence: Nuno Almeida, Gastroenterology Department, Coimbra's University Hospital, Avenida Bissaya Barreto and Praceta Mota Pinto, 3000-075 Coimbra, Portugal. Email: nunoperesalmeida@ gmail.com

Received 7 June 2008; accepted 4 August 2008 mens demonstrated the presence of a brown pigment within macrophages of the lamina propria (Fig. 3) with Perl's reaction confirming it was hemosiderin deposition (Fig. 4).

In both patients, there was no dark pigmentation of the colonic mucosa. They had a normal body mass index and no dietary restrictions.

\section{DISCUSSION}

Melanosis coli is a frequent condition that results from deposition of lipofuscin in the macrophages within the lamina propria. ${ }^{1}$ Duodenal pseudomelanosis is the most reported extracolonic site, but jejunal and ileal pseudomelanosis are reported only occasionally in the literature. ${ }^{2-4}$ With $\mathrm{CE}$ and DBE, accessibility of the small bowel has increased significantly and, so, it is likely that the number of detected cases would increase. However, since the introduction of new enteroscopy methods, only a few cases have been reported. ${ }^{4}$ In our center, there was only one case in more than 600 capsule enteroscopies (more than $25 \%$ with oral iron therapy) and another one in more than 100 double-balloon enteroscopies. A recent retrospective study revealed that histological deposition of pigments might be apparent earlier than the endoscopic detection. ${ }^{5}$ Probably, the real incidence of pseudomelanosis is higher than we think but only systematic biopsies would reveal it.

In small bowel pseudomelanosis, the pigments deposited within macrophages in the lamina propria are essentially iron sulfide and/or hemosiderin but deposition of lipofuscin, silicates, titanium and other minerals is also possible. It seems that there is a strong correlation between small bowel pseudomelanosis and oral iron therapy, ${ }^{4}$ although some authors defend that oral iron intake is not the sole condition associated with pseudomelanosis. In fact, association with hypertension/antihypertensive medications, end-stage renal disease and diabetes mellitus is also possible. ${ }^{5}$ Our second case reinforces the association with iron intake, as no mucosa 


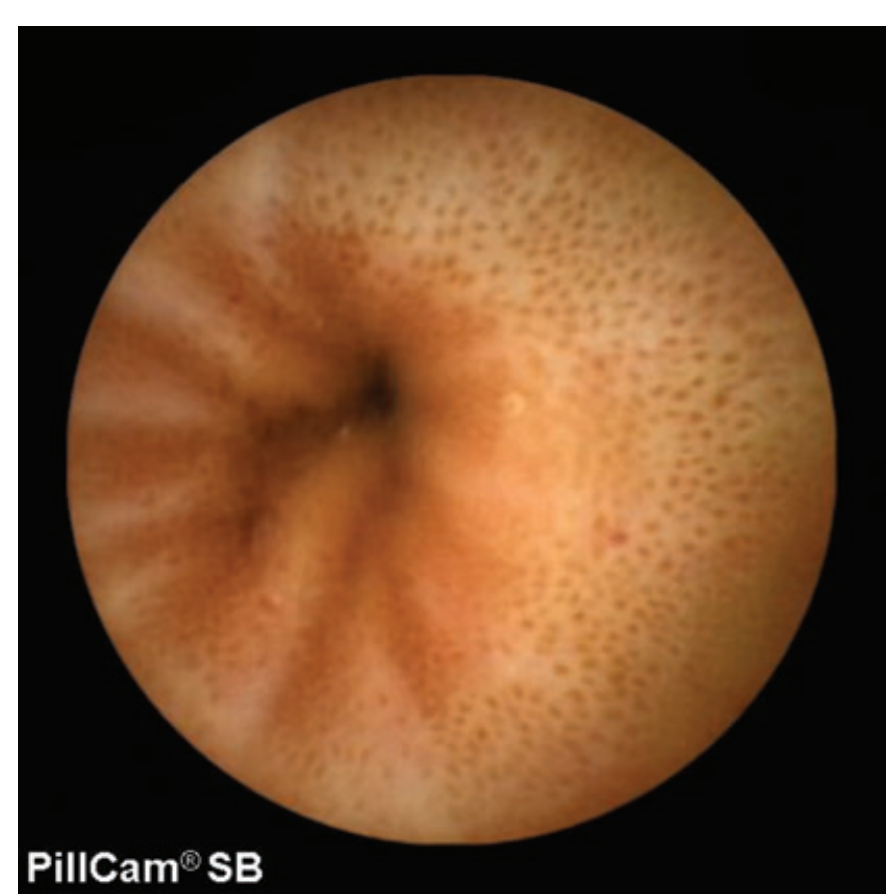

Fig. 1. Capsule endoscopic image shows a brown pigmentation with a speckled, continuous pattern in the proximal jejunum.

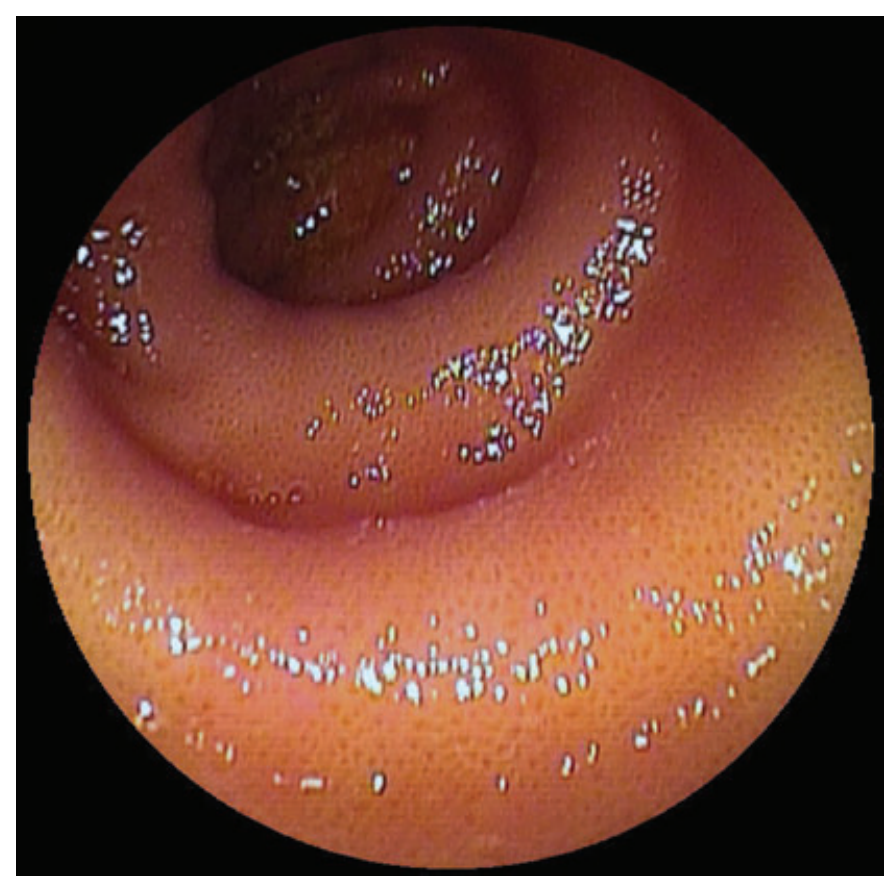

Fig. 2. Double-balloon enteroscopy revealed the same brown pigmentation with a speckled pattern in the distal ileum.

pigmentation was visible at a previous enteroscopy. In the 2 years between both retrograde DBE, the patient maintained oral iron therapy and histological study of biopsy specimens confirmed iron deposition in the macrophages in the lamina propria. However, as reported in other cases, we found no clear mechanisms for pigment deposition. Coupling of absorbed iron with a sulfur moiety from antihypertensive medications could be a hypothesis for pigment accumulation in the macrophages of the lamina propria. ${ }^{5}$

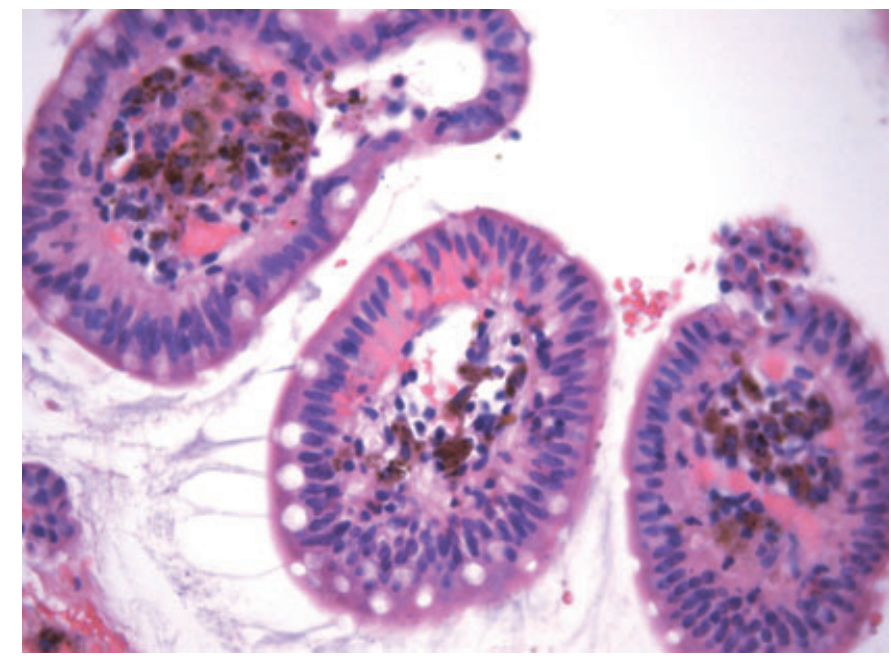

Fig. 3. Distal ileum biopsies revealed abundant hyperpigmented macrophages in the lamina propria $(H \& E, \times 400)$.

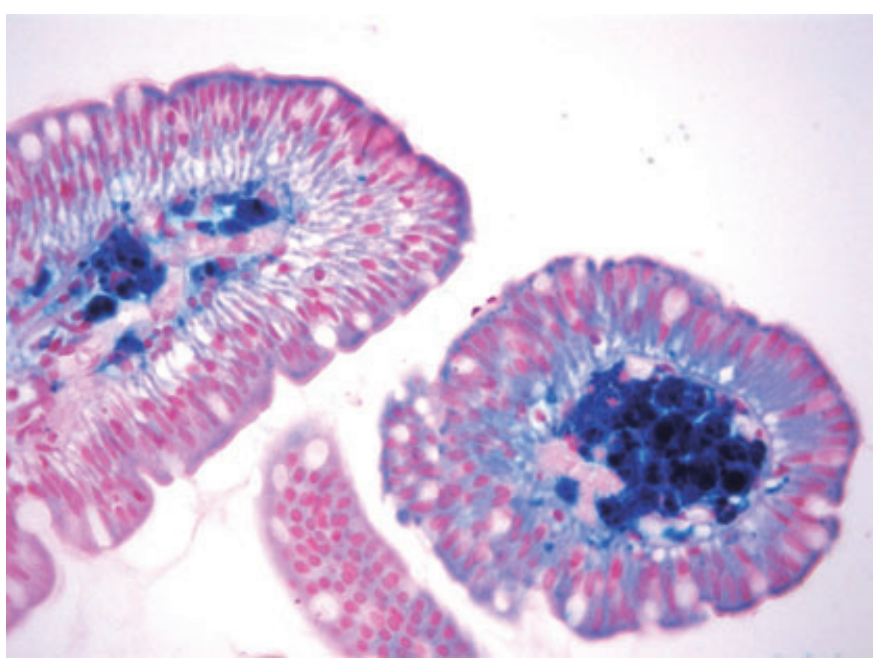

Fig. 4. Intense pigmentation revealing iron deposition as hemosiderin (blue coloration; Perl's prussian blue; $\times 400$ ).

Until now, the diagnostic and prognostic significance of small bowel pseudomelanosis has not been determined and there are no follow-up strategies. ${ }^{5}$ It seems that pseudomelanosis is a histological and/or endoscopic finding without pathological implications.

The findings in these two cases demonstrate the importance of the new enteroscopy methods in the diagnosis of pseudomelanosis and strengthen its possible association with oral iron therapy. However, contrary to pseudomelanosis coli, which occurs in more than $70 \%$ of persons who use anthraquinone laxatives, pseudomelanosis of the small bowel seems to be a very rare event, even in patients on chronic oral iron therapy.

\section{REFERENCES}

1. Wald A. Other diseases of the colon and rectum. In: Feldman M, Friedman LS, Brandt LJ (eds). Sleisenger and Fordtran's Gastrointestinal and Liver Disease, 8th edn. Philadelphia: Elsevier, 2006; 2811-32. 
2. Weinstock L, Shah R. Gastric pseudomelanosis (review). Gastroenterol Hepatol 2006; 2: 806-9.

3. Qutob T, Goldstein J, Ryan C et al. Terminal ileum hemosiderosis in association with hereditary hemochromatosis. $J$. Clin. Gastroenterol. 2004; 38: 465.
4. Moore JD, Baichi M, Toledo R, Sitrin M. Pseudomelanosis of jejunum and ileum. Gastrointest. Endosc. 2007; 66: 857-9.

5. Giusto D., Jakate S. Pseudomelanosis duodeni: Associated with multiple clinical conditions and unpredictable iron stainability - A case series. Endoscopy 2008; 40: 165-7. 